\title{
Hydroxyl safflower yellow B combined with doxorubicin inhibits the proliferation of human breast cancer MCF-7 cells
}

\author{
KEHAO LIN $^{1 *}$, ZE QIN $^{2 *}$, CHUANJUN QU $^{1}$, XIAOYU CHEN ${ }^{1}$, QINGLING JIANG ${ }^{1}$, \\ MINJING LI ${ }^{1}$, QIUSHENG ZHENG ${ }^{1,3}$ and DEFANG LI ${ }^{1}$ \\ ${ }^{1}$ Yantai Key Laboratory of Pharmacology of Traditional Chinese Medicine in Tumor Metabolism, \\ School of Integrated Traditional Chinese and Western Medicine, Binzhou Medical University, Yantai, \\ Shandong 264003; ${ }^{2}$ Department of Anesthesia, The Fourth Hospital of Shijiazhuang, Shijiazhuang, Hebei 050011; \\ ${ }^{3}$ Key Laboratory of Xinjiang Endemic Phytomedicine Resources of Ministry of Education, \\ School of Pharmacy, Shihezi University, Shihezi, Xinjiang 832002, P.R. China
}

Received November 2, 2020; Accepted February 25, 2021

DOI: 10.3892/ol.2021.12687

\begin{abstract}
Doxorubicin (DOX) is currently the preferred chemotherapeutic agent for breast cancer, and hydroxyl safflower yellow B (HSYB) has a tumor growth-inhibiting activity. The present study aimed to investigate the effects of HSYB combined with DOX on the proliferation of human breast cancer MCF-7 cells and explore the underlying mechanism. MTT and cell colony formation assays revealed that the proliferation rate of MCF-7 cells was signifiscantly decreased after HSYB and DOX treatment. Combined HSYB and DOX treatment significantly decreased the expression levels of BCL-2 in MCF-7 cells, while the expression levels of apoptosis-associated proteins, including cleaved caspase-9, BAX and cleaved caspase-3, were markedly increased. Furthermore, flow cytometry and western blot analysis demonstrated that combined HSYB and DOX treatment stimulated an increase in intracellular reactive oxygen species and promoted the release of cytochrome $c$, leading to apoptosis. The current data suggested that the combination of HSYB and DOX may have marked antitumor activity.
\end{abstract}

Correspondence to: Professor Defang $\mathrm{Li}$ or Professor Qiusheng Zheng, Yantai Key Laboratory of Pharmacology of Traditional Chinese Medicine in Tumor Metabolism, School of Integrated Traditional Chinese and Western Medicine, Binzhou Medical University, 346 Guanhai Road, Yantai, Shandong 264003, P.R. China

E-mail: lidefang@163.com

E-mail: zqsyt@sohu.com

*Contributed equally

Key words: hydroxyl safflower yellow B, doxorubicin, breast cancer, apoptosis, MCF-7 cells

\section{Introduction}

Breast cancer is an invasive cancer, ranking first in the incidence of cancer in women; it accounts for $11.6 \%$ of the total cancer incidences, and $\sim 630,000$ women worldwide died of breast cancer in 2018, accounting for $15 \%$ of cancer-associated deaths in women (1). Compared with the global average, Chinese women have a lower incidence of breast cancer and a higher mortality rate, seriously threatening women's health (2). Breast cancer treatment uses a comprehensive treatment model that includes surgery and chemotherapy (3). The high postoperative recurrence rate and post-surgical complications within a short period following breast cancer surgery are significant $(4,5)$. Therefore, increased attention has been focused on further elucidating the pathogenesis of breast cancer to discover and develop novel treatment methods.

Modern medical research has revealed that the formation and growth of tumors are associated with cellular differentiation and apoptosis (6). Apoptosis-induced inhibition of tumor cell proliferation is currently an effective method for treating tumors (7). After numerous years of development, traditional Chinese medicine has identified a set of unique antitumor methods (8). Modern pharmacological studies have demonstrated that traditional Chinese medicine exerts antitumor effects by inhibiting cellular proliferation, inducing apoptosis, regulating cellular signaling pathways and inhibiting multidrug resistance (9-11). Furthermore, traditional Chinese medicine is widely used in chemotherapy and radiation therapy, which can effectively extend the life expectancy of patients and improve the overall efficacy of treatments (12-14).

As a Chinese herbal medicine, safflower promotes blood circulation and removes blood stasis (15). The red flavonoid hydroxyl safflower yellow A (HSYA) is an effective active ingredient in mitigating numerous different types of tumor, such as liver cancer and glioma (16-19). A novel flavonoid, hydroxyl safflower yellow B (HSYB), which is an isomer of HSYA, has been demonstrated to have a well-defined anti-breast cancer effect $(20,21)$. Doxorubicin (DOX), as an anthracycline anticancer drug, is a classic breast cancer 
chemotherapeutic drug with a high sensitivity to cancer cells that inhibits cell proliferation by inducing apoptosis $(22,23)$. Moreover, combination therapies can exert synergistic therapeutic effects. However, to the best of our knowledge, there are currently no studies on the combination of HSYB and DOX for treating breast cancer. Hence, the present study aimed to investigate the potential synergistic effects of combined DOX and HSYB treatment.

\section{Materials and methods}

Chemicals and reagents. HSYB (purity, >95\%) was donated by Dr Tao Weiwei of Nanjing University of Traditional Chinese Medicine (Nanjing, China). DOX hydrochloride (purity, >98\%; cat. no. 113424) was purchased from Beijing Bailingwei Technology Co., Ltd. Dulbecco's modified Eagle's medium (DMEM) was purchased from Thermo Fisher Scientific, Inc. Fetal bovine serum (FBS) was purchased from Zhejiang Tianhang Biotechnology Co., Ltd. Dimethyl sulfoxide (DMSO), Penicillin-Streptomycin, MTT, $0.25 \%$ trypsin digestion solution (without phenol red), Hoechst 33258 staining solution, Annexin V-FITC Apoptosis Detection kit, Reactive Oxygen Species Assay kit and Mitochondrial Membrane Potential Assay kit with JC-1 were ordered from Beijing Solarbio Science \& Technology Co., Ltd. Anti-cleaved caspase-3 (1:1,000; cat. no. 9662), anti-cleaved caspase-9 (1:1,000; cat. no. 9508), anti-cytochrome c (1:1,000; cat. no. 4280) and anti-BAX (1:1,000; cat. no. 3498) were purchased from Cell Signaling Technology, Inc. Anti-Bcl-2 (1:1,000; cat. no. ab196495), anti-caspase-9 (1:1,000; cat. no. ab25758) and anti-caspase-3 (1:500; cat. no. ab13847) were purchased from Abcam. Primary antibodies against $\beta$-actin (1:2,000; cat. no. TA-09) and peroxidase-conjugated goat anti-mouse IgG secondary antibodies (1:25,000; cat.no. ZB2305) were purchased from OriGene Technologies, Inc.

Cell lines and cell culture. MCF-7 cells were obtained from The Cell Bank of Type Culture Collection of the Chinese Academy of Sciences. HaCaT cells were purchased from Procell Life Science \& Technology Co., Ltd.. The cells were cultured at $37^{\circ} \mathrm{C}$ with $5 \% \mathrm{CO}_{2}$ in DMEM with $10 \%$ FBS and $1 \%$ penicillin-streptomycin.

Determination of cellular viability. HaCaT $\left(1.0 \times 10^{4}\right.$ cells/ well) or MCF-7 cells $\left(0.7 \times 10^{4}\right.$ cells/well) in 96 -well plates were treated with DOX $(0.1-2 \mu \mathrm{g} / \mathrm{ml})$, HSYB $(5-30 \mu \mathrm{g} / \mathrm{ml})$ or a combination of the two for $24 \mathrm{~h}$ at $37^{\circ} \mathrm{C}$. Subsequently, MTT $(20 \mu \mathrm{l})$ was added to each well and incubated for $4 \mathrm{~h}$ at $37^{\circ} \mathrm{C}$. DMSO $(150 \mu \mathrm{l})$ was then directly added to the plate while shaking at low speed for $10 \mathrm{~min}$ at room temperature. Finally, the optical density (OD) of the cells at $490 \mathrm{~nm}$ was determined using an enzyme-labeling instrument, and the inhibition rate was calculated as follows: (1-OD value of experimental group/OD value of control group) x $100 \%$. In addition, according to the Chou-Talalay combination index (CI) method (24), the CI was calculated to evaluate the effect of drug combination and to test the interaction between two drugs with different concentrations. Data were analyzed using Compusyn 2.0 software (Biosoft) to calculate CI and Fa values. $\mathrm{CI}<1, \mathrm{CI}=1$ and $\mathrm{CI}>1$ indicated synergistic, additive and antagonistic effects, respectively.

Colony formation experiments. MCF-7 cells were inoculated into a 6 -well plate $(200$ cells/well) and treated with DOX $(0.4 \mu \mathrm{g} / \mathrm{ml})$, HSYB $(6 \mu \mathrm{g} / \mathrm{ml})$ or a combination of the two for $24 \mathrm{~h}$ at $37^{\circ} \mathrm{C}$ after attachment. The complete fresh medium was replaced every three days. After $\sim 12$ days of culture, the cells were fixed at room temperature with $4 \%$ paraformaldehyde solution for $20 \mathrm{~min}$. Subsequently, the cells were washed with PBS and treated with crystal-violet staining solution for $10 \mathrm{~min}$ at room temperature. Finally, the dye was cleaned with PBS, and the stained cells were imaged using a camera. A colony was defined as $>50$ cells.

Hoechst 33258 staining detection. An aseptic cover slide was placed into a 6-well plate and inoculated with MCF-7 cells $\left(1.4 \times 10^{5}\right.$ cells/well). Cells were fixed with $4 \%$ paraformaldehyde for $20 \mathrm{~min}$ at room temperature after treatment with DOX $(0.4 \mu \mathrm{g} / \mathrm{ml})$, HSYB $(6 \mu \mathrm{g} / \mathrm{ml})$ or a combination of the two for $24 \mathrm{~h}$ at $37^{\circ} \mathrm{C}$. Subsequently, the cells were washed with PBS and then stained with Hoechst 33258 for 10 min at room temperature. The staining solution was cleaned with PBS, and the slides were observed under an inverted fluorescence microscope (magnification, x400).

Annexin V-FITC apoptosis detection. MCF-7 cells ( $1.0 \times 10^{6}$ cells/group) treated with DOX $(0.4 \mu \mathrm{g} / \mathrm{ml})$, HSYB $(6 \mu \mathrm{g} / \mathrm{ml})$ or a combination of the two drugs were collected and resuspended in a staining buffer (500 $\mu \mathrm{l})$ containing Annexin V-FITC $(5 \mu 1)$, after which they were tested using a FACSCanto II flow cytometer (Becton, Dickinson and Company). The apoptosis rate was analyzed using the FACSDiva 6.1.3 software (Becton, Dickinson and Company).

Western blot analysis. MCF-7 cells (5.7×10 6 cells/well) were seeded and treated as aforementioned in Petri dishes (100-mm). Protein was extracted using RIPA lysis buffer (cat. no. R0010; Beijing Solarbio Science \& Technology Co., Ltd.) after the cells were collected. The protein concentration was detected using a BCA protein assay kit (cat. no. PC0020; Beijing Solarbio Science $\&$ Technology Co., Ltd.). Proteins ( $80 \mu \mathrm{g} /$ lane) were separated via $12 \%$ SDS-PAGE and transferred to a PVDF membrane. Protein bands were blocked with 5\% BSA (cat. no. 232100; Becton, Dickinson and Company) at room temperature for $3 \mathrm{~h}$ and were then incubated overnight with primary antibodies at $4^{\circ} \mathrm{C}$. The samples were washed four times with TBS-Tween (TBST; $0.1 \%$ of Tween-20) buffer (6 min per wash), incubated with secondary antibodies for $35 \mathrm{~min}$ at room temperature and washed four times with TBST buffer (6 min per wash). Finally, the protein bands were incubated with an ECL chemiluminescence working buffer (cat. no. 34580; Thermo Fisher Scientific, Inc.), and western blotting was imaged via a UVP imaging system. ImageJ v1.43 software (National Institutes of Health) was used for densitometry.

Detection of reactive oxygen species (ROS) in cells. MCF-7 cells $\left(1.8 \times 10^{5}\right.$ cells/well) were plated in Petri dishes $(30-\mathrm{mm})$. Cells were treated with DOX $(0.4 \mu \mathrm{g} / \mathrm{ml})$, HSYB $(6 \mu \mathrm{g} / \mathrm{ml})$ or a combination of the two for $24 \mathrm{~h}$ at $37^{\circ} \mathrm{C}$. The cells were 

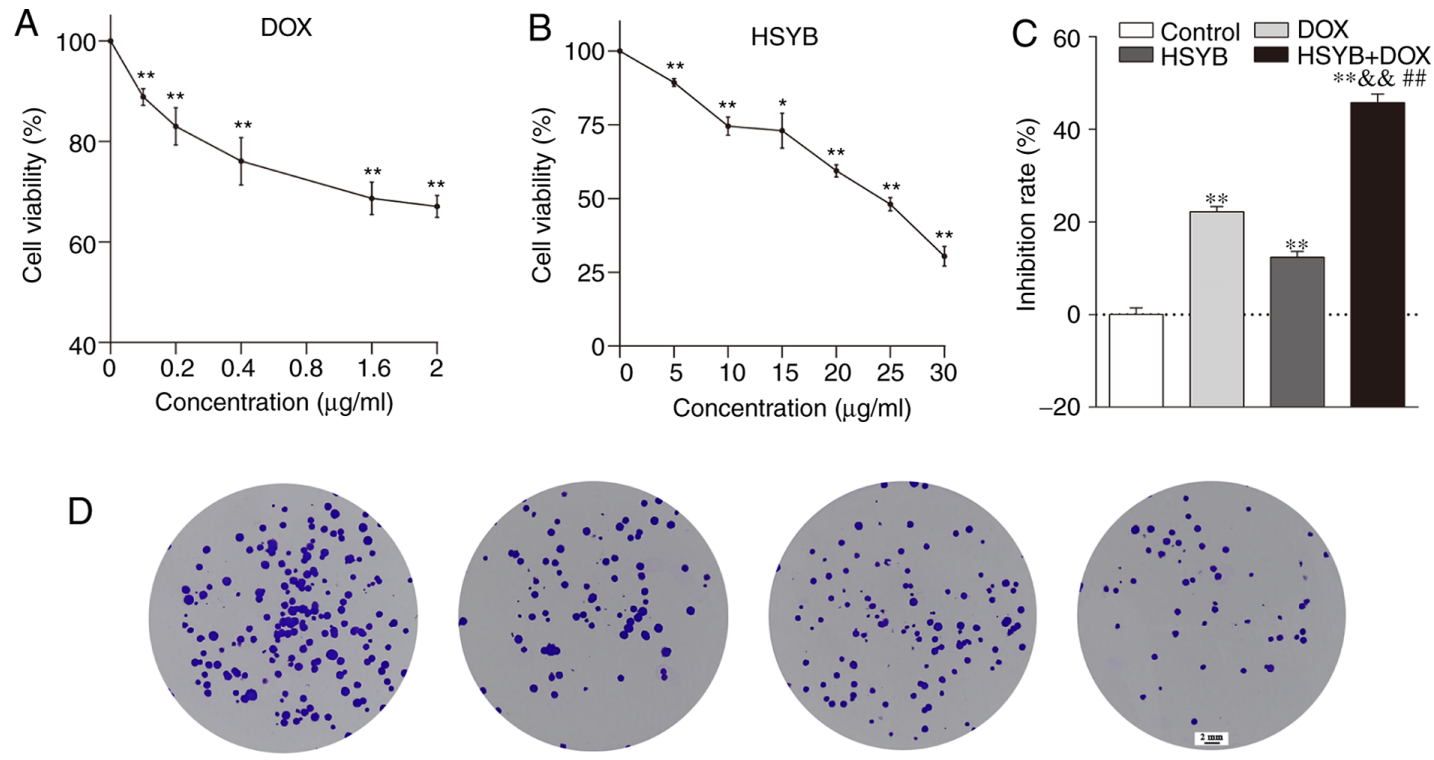

$\operatorname{DOX}(\mu \mathrm{g} / \mathrm{ml})$

$\operatorname{HSYB}(\mu \mathrm{g} / \mathrm{ml})$

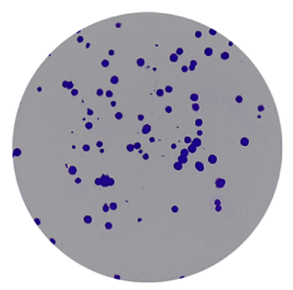

0.4

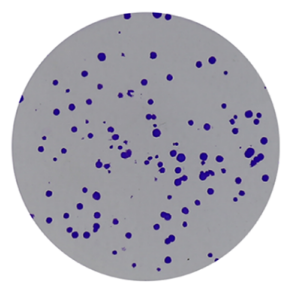

$-$

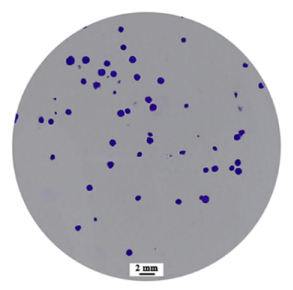

0.4

6

Figure 1. Combination of HSYB and DOX effectively inhibits the proliferation of breast cancer MCF-7 cells. Cellular viability was measured via the MTT method after $24 \mathrm{~h}$ of (A) DOX and (B) HSYB treatment at different concentrations. (C) Inhibition rate of DOX (0.4 $\mu \mathrm{g} / \mathrm{ml}), \mathrm{HSYB}(6 \mu \mathrm{g} / \mathrm{ml})$ or DOX $+\mathrm{HSYB}$ combination treatment for $24 \mathrm{~h}$. (D) Representative images of colony formation assays after DOX $(0.4 \mu \mathrm{g} / \mathrm{ml}), \mathrm{HSYB}(6 \mu \mathrm{g} / \mathrm{ml})$ or DOX $+\mathrm{HSYB}$ treatment for $24 \mathrm{~h}$. Data are presented as the mean $\pm \mathrm{SD}(\mathrm{n}=3)$. ${ }^{*} \mathrm{P}<0.05$ and ${ }^{* *} \mathrm{P}<0.01$ vs. control; \&\& $\mathrm{P}<0.01$ vs. DOX; ${ }^{\# \#} \mathrm{P}<0.01$ vs. HSYB. DOX, doxorubicin; HSYB, hydroxyl safflower yellow B.

detached from the plate with EDTA-containing trypsin and resuspended in serum-free medium containing a DCFH-DA probe $(10 \mu \mathrm{mol} / \mathrm{l})$. Subsequently, the cells were transferred to a thermostatic chamber at $37^{\circ} \mathrm{C}$ for $20 \mathrm{~min}$. Finally, the cells were washed three times with TBST buffer, and the DCF fluorescence intensity of the treated cells was determined using a FACSCanto II flow cytometer. The DCF fluorescence intensity was analyzed using the FACSDiva 6.1.3 software (Becton, Dickinson and Company). In addition, aseptic cover slides were placed into a 6-well plate and inoculated cells were imaged under an inverted fluorescence microscope (magnification, x200). The dye solutions used for ROS detection were from a Reactive Oxygen Species Assay kit (cat. no. CA1410; Beijing Solarbio Science \& Technology Co., Ltd.).

Measurement of mitochondrial membrane potential (MMP). As aforementioned, DOX- and HSYB-treated cells were treated with EDTA-containing trypsin. The cells were resuspended in complete medium $(500 \mu \mathrm{l})$ containing JC-1 staining working solution $(500 \mu \mathrm{l})$ and then incubated at $37^{\circ} \mathrm{C}$ for $20 \mathrm{~min}$. Next, cells were washed twice with the JC-1 staining buffer (1X). Finally, the JC-1 staining buffer (500 $\mu \mathrm{l})$ was used to resuspend the cells, which were subsequently analyzed using a FACSCanto II flow cytometer. The ratio of red to green fluorescence was analyzed using the FACSDiva 6.1.3 software.

Statistical analysis. The SPSS 21.0 software (IBM Corp.) was used to analyze the data presented as the mean \pm SD. Significant differences were determined using an unpaired Student's t-tests or one-way ANOVA followed by Bonferroni's post hoc test. $\mathrm{P}<0.05$ was considered to indicate a statistically significant difference.

\section{Results}

Combination of HSYB and DOX has a synergistic effect on MCF-7 cells. The viability of HSYB and/or DOX-treated MCF-7 cells was assessed using the MTT method to investigate whether there was a synergistic effect of HSYB and DOX. First, MCF-7 cells were incubated with different concentrations of HSYB $(0-30 \mu \mathrm{g} / \mathrm{ml})$ or DOX $(0-2 \mu \mathrm{g} / \mathrm{ml})$ for $24 \mathrm{~h}$. The inhibitory effect of HSYB and DOX on breast cancer MCF-7 cells was significantly decreased in a concentration-dependent manner (Fig. 1A and B). The $\mathrm{IC}_{50}$ values of HSYB and DOX were $22.12 \mu \mathrm{g} / \mathrm{ml}$ and $6.85 \mu \mathrm{g} / \mathrm{ml}$, respectively, after $24 \mathrm{~h}$ of drug administration. Subsequently, the effect of the combination of HSYB and DOX for $24 \mathrm{~h}$ on the viability of MCF-7 cells was detected via MTT assays. The Chou-Talalay method and Compusyn 2.0 software were used to calculate the combination index (CI). The present results demonstrated that HSYB synergistically enhanced the efficacy of DOX against breast cancer MCF-7 cells. When HSYB $(6 \mu \mathrm{g} / \mathrm{ml})$ was combined with $\operatorname{DOX}(0.4 \mu \mathrm{g} / \mathrm{ml})$, the CI value was the smallest, and the effect of coadministration was the most obvious $(\mathrm{CI}<1$; Table SI). Therefore, these concentrations were used for subsequent experiments. Compared with HSYB $(6 \mu \mathrm{g} / \mathrm{ml})$ or DOX $(0.4 \mu \mathrm{g} / \mathrm{ml})$ alone, inhibition of cellular proliferation was significantly enhanced after combined treatment with HSYB and DOX (Fig. 1C).

Furthermore, the effects of HSYB, DOX or combination therapy on cell colony formation were studied. There were fewer cell colonies in the DOX or HSYB groups than in the 
A

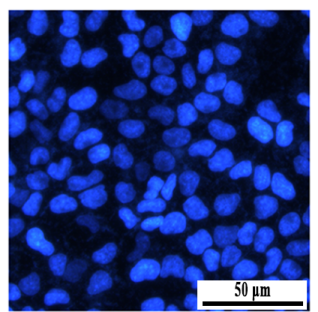

$\operatorname{DOX}(\mu \mathrm{g} / \mathrm{ml})$

$\operatorname{HSYB}(\mu \mathrm{g} / \mathrm{ml})$

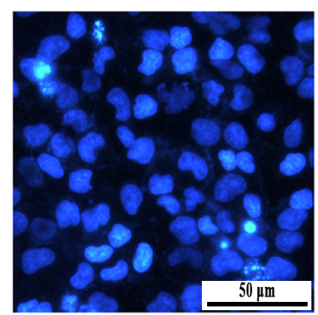

0.4

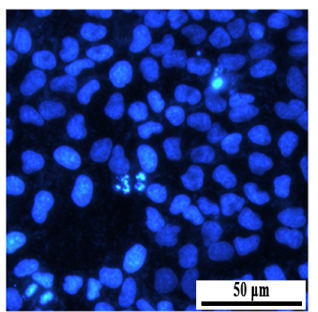

$-$

6

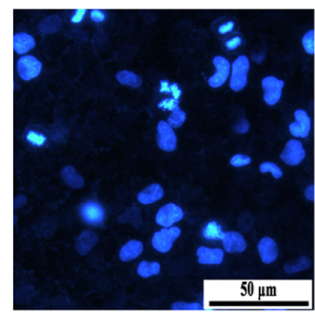

0.4

6
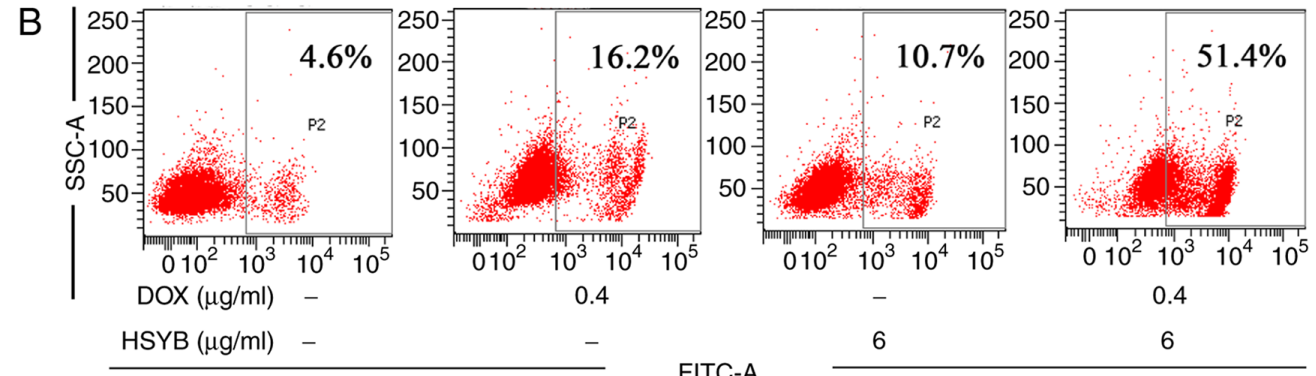

FITC-A

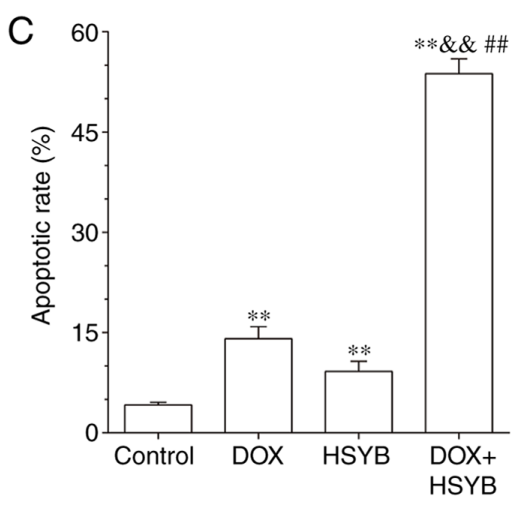

Figure 2. Effects of combination of HSYB and DOX on apoptosis in breast cancer MCF-7 cells. (A) Morphological changes of cells were detected by Hoechst staining. Scale bar, $50 \mu \mathrm{m}$. (B) After Annexin V-FITC staining, flow cytometry was used to detect apoptosis in different groups. (C) Quantitative graph of MCF-7 cell apoptotic rate. Data are presented as the mean $\pm \mathrm{SD}(\mathrm{n}=3){ }^{*}{ }^{* *} \mathrm{P}<0.01 \mathrm{vs}$. control; ${ }^{\& \&} \mathrm{P}<0.01 \mathrm{vs}$. DOX; ${ }^{\# /} \mathrm{P}<0.01 \mathrm{vs}$. HSYB. DOX, doxorubicin; HSYB, hydroxyl safflower yellow B; SSC, side scatter.

untreated (control) group, and after combined HSYB and DOX treatment, the number of cell colonies was markedly decreased (Fig. 1D). Therefore, the combination of HSYB and DOX enhanced the inhibition of MCF-7 cell proliferation. In addition, the normal human keratinocyte $\mathrm{HaCaT}$ cell line was chosen as a control group. The results revealed that DOX $(0.1-2 \mu \mathrm{g} / \mathrm{ml})$ significantly inhibited the proliferation of $\mathrm{HaCaT}$ cells and had a higher cytotoxicity in $\mathrm{HaCaT}$ cells than in MCF-7 cells (Fig. S1A). Additionally, the results revealed that a high concentration $(25 \mu \mathrm{g} / \mathrm{ml})$ of HSYB significantly inhibited the proliferation of $\mathrm{HaCaT}$ cells and had a higher cytotoxicity in these cells than in MCF-7 cells; however, a low concentration $(5 \mu \mathrm{g} / \mathrm{ml})$ of HSYB significantly promoted the proliferation of $\mathrm{HaCaT}$ cells (Fig. S1B). Based on these data, a lower concentration $(6 \mu \mathrm{g} / \mathrm{ml})$ of HSYB was selected for the combined treatment with HSYB and DOX.

Combination of HSYB and DOX induces apoptosis in MCF-7 cells. The synergistic effects of HSYB and DOX on MCF-7 apoptosis and cellular morphology were further investigated. Using Hoechst 33258 staining, it was revealed that the HSYB and DOX combined-treatment group exhibited typical apoptotic morphological characteristics compared with the control and the single drug groups, with the combined therapy group exhibiting a higher concentration of nuclear chromatin and the formation of apoptotic bodies (Fig. 2A).

The apoptotic rate of the HSYB and DOX combined- treatment group in MCF-7 cells was further evaluated by Annexin V-FITC staining. Treatment with DOX or HSYB alone significantly increased apoptosis compared with the control group, but the combined use of DOX and HSYB further significantly increased apoptosis (Fig. 2B and C). The apoptotic rate of MCF-7 cells increased from 4.2 to $14.1,9.2$ and $53.7 \%$ after treatment with DOX, HSYB and a combination of HSYB and DOX, respectively (Fig. 2C). Therefore, the present results confirmed that DOX combined with HSYB significantly induced apoptosis of MCF-7 cells.

Combination of HSYB and DOX affects the level of apoptosis-associated molecular proteins in MCF-7 cells. Western blotting was employed to identify the synergistic effects of HSYB and DOX on the expression levels of signaling 

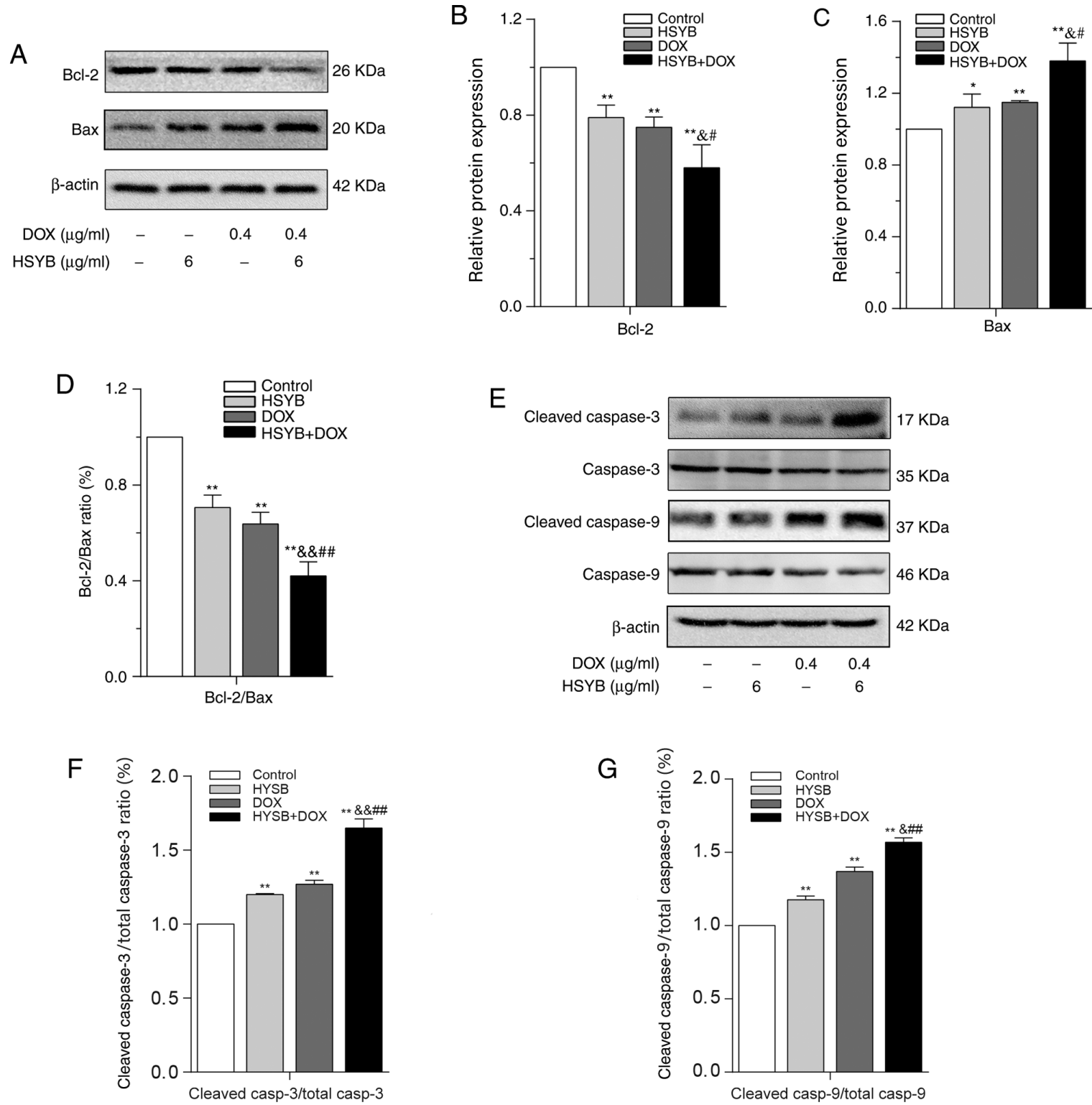

Figure 3. Effects of combination of HSYB and DOX on the expression levels of apoptosis-associated molecular proteins in breast cancer MCF-7 cells. (A) BAX and BCL-2 protein expression were measured by western blotting. Quantitative graph of protein levels of (B) BCL-2, (C) BAX and (D) BCL-2/BAX in MCF-7 cells. (E) Protein expression levels of caspase-3, cleaved caspase-3, caspase-9 and cleaved caspase-9 were measured by western blotting. Quantitative graphs of protein expression levels of (F) cleaved caspase-3/total caspase-3 and (G) cleaved caspase-9/total caspase-9 in MCF-7 cells. Data are presented as the mean $\pm \mathrm{SD}(\mathrm{n}=3) .{ }^{* *} \mathrm{P}<0.01$ vs. control; ${ }^{\&} \mathrm{P}<0.05$ and ${ }^{\& \&} \mathrm{P}<0.01$ vs. DOX; ${ }^{*} \mathrm{P}<0.05$ and ${ }^{\# \#} \mathrm{P}<0.01$ vs. HSYB. DOX, doxorubicin; HSYB, hydroxyl safflower yellow B.

molecules associated with apoptosis in MCF-7 cells. BAX expression in MCF-7 cells in the single drug groups was significantly upregulated compared with in the control group, while the levels of BCL-2 and BCL-2/BAX were significantly decreased, and this effect was even more marked in the HSYB and DOX combined-treatment group (Fig. 3A-D). Further research revealed that the ratios of cleaved caspase- $3 /$ total caspase- 3 and cleaved caspase- $9 /$ total caspase- 9 in the HSYB and DOX combined-treatment group were significantly higher compared with in the untreated and single drug groups (Fig. 3E-G). These findings demonstrated that a combination of HSYB and DOX had the strongest regulatory effect on apoptotic proteins, indicating that a combination of HSYB and DOX synergistically induced apoptosis of MCF-7 cells.
Combination of HSYB and DOX increases ROS levels in MCF-7 cells and activates the mitochondrial apoptotic pathway. To further determine whether the synergistic effects of HSYB and DOX in inducing apoptosis of MCF-7 cells were associated with ROS and the mitochondrial apoptotic pathway, ROS levels were detected by DCFH-DA staining and the MMP by JC-1 staining. The ROS levels of MCF-7 cells treated with HSYB and/or DOX were higher than in control cells, and the combined-treatment group exhibited the highest ROS levels (Fig. 4A and B). Furthermore, MMP was significantly lower in cells treated with HSYB and/or DOX than in the control group, and the combined-treatment group had the most pronounced effect (Fig. 4C and D). Additionally, the synergistic effects of HSYB and DOX on cytoplasmic cytochrome $c$ (Cyto-c) levels in MCF-7 cells 


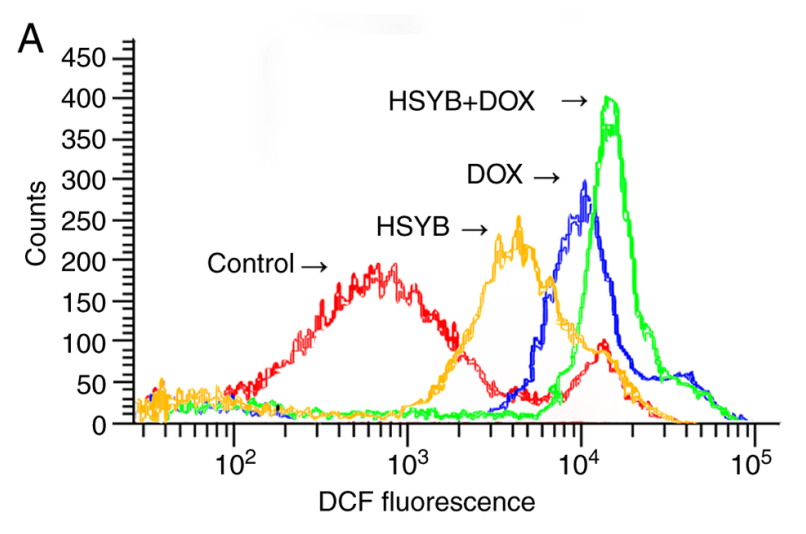

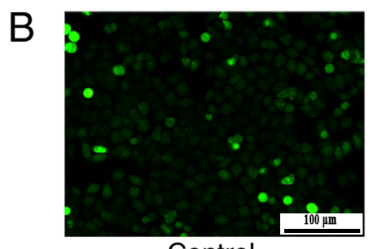

Control

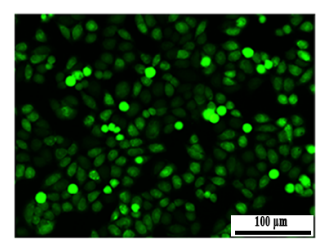

DOX
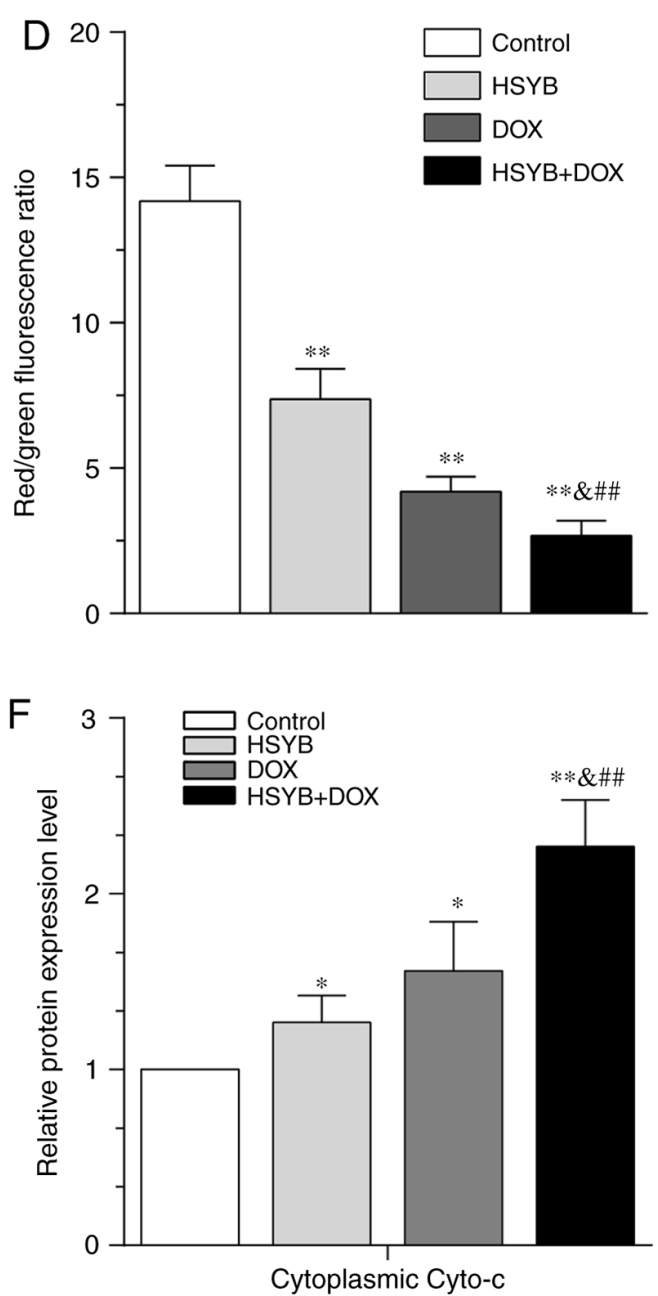

Figure 4. Effects of combination of HSYB and DOX on ROS levels in breast cancer MCF-7 cells. (A) ROS levels in MCF-7 cells were detected by flow cytometry. (B) Representative images of changes in intracellular ROS levels. Scale bar, $100 \mu \mathrm{m}$. (C) Changes in mitochondrial membrane potential in MCF-7 cells were detected by flow cytometry. (D) Quantitative graph of JC-1 red and green fluorescence ratios. (E) Cytoplasmic Cyto- $c$ protein levels were measured by western blotting. (F) Quantitative graph of Cyto-c protein levels in MCF-7 cells. Data are presented as the mean $\pm \mathrm{SD}(\mathrm{n}=3)$. " $\mathrm{P}<0.05$ and ${ }^{* * *} \mathrm{P}<0.01$ vs. control; ${ }^{\circledR} \mathrm{P}<0.05$ vs. DOX; ${ }^{\# /} \mathrm{P}<0.01$ vs. HSYB. DOX, doxorubicin; HSYB, hydroxyl safflower yellow B; ROS, reactive oxygen species; Cyto-c, cytochrome $c$.

were detected by western blotting. It was revealed that the Cyto-c levels of MCF-7 cells in the single drug groups were higher than in the control group, with a further significant increase in the combined-treatment group (Fig. 4E and F). The current results confirmed that a combination of HSYB and DOX promoted ROS levels in MCF-7 cells and activated the mitochondrial apoptotic pathway.

\section{Discussion}

In most countries globally, breast cancer has the highest incidence and mortality rates among female-specific types of cancer (1). DOX represents the first-line drug for breast cancer treatment; however, it also induces side effects, such as cardiac toxicity $(25,26)$. Safflower can be used for treating 
angina pectoris or cardiovascular/cerebrovascular diseases, and the effective component, HSYA, exerts antitumor effects $(18,27,28)$. Recently, combinations of different drugs have been widely used to treat tumors and have yielded effective results (29). A number of studies have obtained encouraging results on the combination of Chinese herbal medicine and chemotherapeutic drugs to treat tumors, indicating that traditional Chinese medicine may be a safe and effective auxiliary approach for tumor treatment (13,30-32). The present study revealed that HSYB, a flavonoid component in safflower, combined with DOX significantly inhibited breast cancer MCF-7 cell proliferation.

Apoptosis is a form of programmed cell death that requires the participation of various complex factors and is an indispensable component in multicellular life processes $(33,34)$. Numerous studies have demonstrated that apoptosis is affected by various apoptosis-associated factors, including BCL-2 $(35,36)$. Furthermore, BCL-2 and BAX serve opposite roles in the process of apoptosis (37). BCL-2 inhibits apoptosis and promotes cell proliferation $(38,39)$ while BAX is mostly distributed in the cytoplasm, it can destroy the integrity of the mitochondrial membrane and promote apoptosis after being activated (40-42).

Normally, BCL-2 expression is relatively stable (43). When BAX is highly expressed, numerous BAX/BAX homodimers are formed to induce apoptosis (44). By contrast, when BCL-2 expression is upregulated, BCL-2/BAX heterodimers are formed (45). Many BAX/BAX homodimers are dissociated, decreasing the responsiveness of cells to apoptotic signals, which inhibits apoptosis (46). Therefore, the ratio between these two antagonistic proteins is key to cell survival $(44,46)$. With the decrease in BCL-2/BAX levels, the permeability of the mitochondrial membrane is altered, Cyto-c and apoptosis-promoting factors are released into the cytoplasm, and the apoptotic promoter caspase- 9 is activated, directly promoting apoptosis by activating the downstream effector caspase- $3(47,48)$. In the present study, the BCL-2/BAX ratio was significantly decreased, the level of Cyto- $c$ was increased and the levels of the apoptotic promoter (cleaved caspase-9) and the apoptotic effector (cleaved caspase-3) were also upregulated after combined treatment with HSYB and DOX.

ROS are closely associated with the induction and regulation of apoptosis. Increased intracellular ROS levels can cause cellular damage, alter mitochondrial membrane permeability and decrease MMP, thereby promoting Cyto- $c$ release into the cytoplasm and inducing apoptosis (49,50). In the present study, the combination of HSYB and DOX in MCF-7 cells increased ROS levels and decreased the MMP, as determined by fluorescence microscopy and flow cytometry. Overall, the current results revealed that a combination of HSYB and DOX synergistically induced apoptosis of MCF-7 cells by increasing ROS levels, downregulating BCL-2/BAX ratio, promoting Cyto-c release into the cytoplasm and subsequently activating caspase-3 and caspase-9.

However, the present study presents some limitations. First, the antitumor experiments testing HSYB and DOX were only performed in a single cell line, the human breast cancer MCF-7 cell line. Therefore, future studies should investigate the effects of HSYB and DOX in other breast cancer cells in vitro and in mouse models in vivo. Second, the effects of the combination of HSYB and DOX on MCF-7 cell invasion and migration were not assessed, which will require further investigation in future studies.

In conclusion, the current study revealed that the combination of HSYB and DOX synergistically induced apoptosis and enhanced antitumor effects in breast cancer MCF-7 cells, indicating that the combination of HSYB and DOX may be a new alternative for clinical treatment of breast cancer.

\section{Acknowledgements}

Not applicable.

\section{Funding}

The present study was funded by the National Natural Science Foundation of China (grant no. 31870338), the Key Research and Development Program of Shandong Province of China (grant no. 2019GSF108214), Taishan Scholars Construction Engineering of Shandong Province (grant no. tsqn201812099), the Dominant Disciplines' Talent Team Development Scheme of Higher Education of Shandong Province (grant no. 2016052410), the Introduction and Cultivation Project for Young Creative Talents of Higher Education of Shandong Province (grant no. 20191008189) and the Guidance Plan of Science and Technology Research and Development of Shijiazhuang (grant no. 171561533).

\section{Availability of data and materials}

All data generated or analyzed during this study are included in this published article.

\section{Authors' contributions}

KL, ZQ, QZ and DL designed the experiments. KL and DL obtained the experimental data and wrote the manuscript. KL and CQ collected cell samples for Hoechst 33258 staining, apoptosis and western blot analysis. CQ, XC, QJ and ML interpreted and analyzed the data. KL and QJ confirm the authenticity of all the raw data. All authors read and approved the final manuscript.

\section{Ethics approval and consent to participate}

Not applicable.

\section{Patient consent for publication}

Not applicable.

\section{Competing interests}

The authors declare that they have no competing interests.

\section{References}

1. Bray F, Ferlay J, Soerjomataram I, Siegel RL, Torre LA and Jemal A: Global cancer statistics 2018: GLOBOCAN estimates of incidence and mortality worldwide for 36 cancers in 185 countries. CA Cancer J Clin 68: 394-424, 2018. 
2. Song QK, Wang XL, Zhou XN, Yang HB, Li YC, Wu JP, Ren J and Lyerly HK: Breast cancer challenges and screening in China: Lessons from current registry data and population screening studies. Oncologist 20: 773-779, 2015.

3. Gradishar WJ, Anderson BO, Abraham J, Aft R, Agnese D, Allison KH, Blair SL, Burstein HJ, Dang C, Elias AD, et al: Breast cancer, version 3.2020, NCCN clinical practice guidelines in oncology. J Natl Compr Canc Netw 18: 452-478, 2020.

4. Lissoni P, Sormani AL, Tancini G, Cattaneo G, Archili C, Mandelli D, Crispino S, Paolorossi F and Barni S: Postoperative hyperprolactinaemia and early recurrence rate in breast cancer. Eur J Cancer 26: 953-956, 1990.

5. Murthy BL, Thomson CS, Dodwell D, Shenoy H, Mikeljevic JS, Forman D and Horgan K: Postoperative wound complications and systemic recurrence in breast cancer. Br J Cancer 97: 1211-1217, 2007

6. Hanahan D and Weinberg RA: Hallmarks of cancer: The next generation. Cell 144: 646-674, 2011.

7. Macherey S, Mallmann P, Malter W, Doerr F, Heldwein M, Wahlers T and Hekmat K: Lung metastasectomy for pulmonary metastatic breast carcinoma. Geburtshilfe Frauenheilkd 77: 645-650, 2017

8. Ling CQ, Yue XQ and Ling C: Three advantages of using traditional Chinese medicine to prevent and treat tumor. J Integr Med 12: 331-335, 2014.

9. Liu X, Li M, Wang X, Dang Z, Yu L, Wang X, Jiang Y and Yang Z: Effects of adjuvant traditional Chinese medicine therapy on long-term survival in patients with hepatocellular carcinoma. Phytomedicine 62: 152930, 2019.

10. Bing Z, Cheng Z, Shi D, Liu X, Tian J, Yao X, Zhang J, Wang Y and Yang K: Investigate the mechanisms of Chinese medicine Fuzhengkangai towards EGFR mutation-positive lung adenocarcinomas by network pharmacology. BMC Complement Altern Med 18: 293, 2018

11. Gezici S and Şekeroğlu N: Current perspectives in the application of medicinal plants against cancer: Novel therapeutic agents. Anticancer Agents Med Chem 19: 101-111, 2019.

12. Meng MB, Wen QL, Cui YL, She B and Zhang RM: Meta-analysis: Traditional Chinese medicine for improving immune response in patients with unresectable hepatocellular carcinoma after transcatheter arterial chemoembolization. Explore (NY) 7: 37-43, 2011.

13. Guo H, Liu JX, Xu L, Madebo T and Baak JP: Traditional Chinese medicine herbal treatment may have a relevant impact on the prognosis of patients with stage IV adenocarcinoma of the lung treated with platinum-based chemotherapy or combined targeted therapy and chemotherapy. Integr Cancer Ther 10: 127-137, 2011

14. Deng LJ, Hu LP, Peng QL, Yang XL, Bai LL, Yiu A, Li Y, Tian HY, Ye WC and Zhang DM: Hellebrigenin induces cell cycle arrest and apoptosis in human hepatocellular carcinoma HepG2 cells through inhibition of Akt. Chem Biol Interact 219: 184-194, 2014.

15. Kim I, Bae J and Kim BJ: Carthami flos regulates gastrointestinal motility functions. Integr Med Res 6: 404-408, 2017.

16. George VC, Dellaire G and Rupasinghe HPV: Plant flavonoids in cancer chemoprevention: Role in genome stability. J Nutr Biochem 45: 1-14, 2017.

17. Yang F, Li J, Zhu J, Wang D, Chen S and Bai X: Hydroxysafflor yellow A inhibits angiogenesis of hepatocellular carcinoma via blocking ERK/MAPK and NF- $\kappa \mathrm{B}$ signaling pathway in $\mathrm{H} 22$ tumor-bearing mice. Eur J Pharmacol 754: 105-114, 2015.

18. Yang J, Wang Y and Guo ML: Identification and mapping of a novel hydroxysafflor yellow A (HSYA) biosynthetic gene in Carthamus tinctorius. Biochem Genet 49: 410-415, 2011

19. Ao H, Feng W and Peng C: Hydroxysafflor yellow A: A promising therapeutic agent for a broad spectrum of diseases. Evid Based Complement Alternat Med 2018: 8259280, 2018.

20. Qu C, Zhu W, Dong K, Pan Z, Chen Y, Chen X, Liu X, Xu W, Lin $\mathrm{H}$, Zheng Q and Li D: Inhibitory effect of hydroxysafflor yellow B on the proliferation of human breast cancer MCF-7 cells. Recent Pat Anticancer Drug Discov 14: 187-197, 2019.

21. Yue S, Tang Y, Xu C, Li S, Zhu Y and Duan JA: Two new quinochalcone C-glycosides from the florets of Carthamus tinctorius Int J Mol Sci 15: 16760-16771, 2014

22. Bouchalova K, Cizkova M, Cwiertka K, Trojanec R and Hajduch M: Triple negative breast cancer-current status and prospective targeted treatment based on HER1 (EGFR), TOP2A and C-MYC gene assessment. Biomed Pap Med Fac Univ Palacky Olomouc Czech Repub 153: 13-17, 2009.
23. Ku JM, Kim SR, Hong SH, Choi HS, Seo HS, Shin YC and Ko SG: Cucurbitacin D induces cell cycle arrest and apoptosis by inhibiting STAT3 and NF- $\mathrm{NB}$ signaling in doxorubicinresistant human breast carcinoma (MCF7/ADR) cells. Mol Cell Biochem 409: 33-43, 2015.

24. Chou TC: Drug combination studies and their synergy quantification using the Chou-Talalay method. Cancer Res 70: 440-446, 2010.

25. Ren B, Ye L, Gong J, Ren H, Ding Y, Chen X, Liu X, Lu P, Wei F $\mathrm{Xu} \mathrm{W}$, et al: Alteronol enhances the anti-tumor activity and reduces the toxicity of high-dose adriamycin in breast cancer. Front Pharmacol 10: 285, 2019.

26. Menna P, Paz OG, Chello M, Covino E, Salvatorelli E and Minotti G: Anthracycline cardiotoxicity. Expert Opin Drug Saf 11 (Suppl 1): S21-S36, 2012.

27. Choi SH, Lee AY, Park CH, Shin YS and Cho EJ: Protective effect of Carthamus tinctorius L. seed on oxidative stress and cognitive impairment induced by chronic alcohol consumption in mice. Food Sci Biotechnol 27: 1475-1484, 2018.

28. Xi S, Zhang Q, Xie H, Liu L, Liu C, Gao X, Zhang J, Wu L, Qian L and Deng X: Effects of hydroxy safflor yellow A on blood vessel and mRNA expression with VEGF and bFGF of transplantation tumor with gastric adenocarcinoma cell line BGC-823 in nude mice. Zhongguo Zhong Yao Za Zhi 34: 605-610, 2009 (In Chinese).

29. Zheng Z, Zhu W, Yang B, Chai R, Liu T, Li F, Ren G, Ji S, Liu S and $\mathrm{Li} \mathrm{G}$ : The co-treatment of metformin with flavone synergistically induces apoptosis through inhibition of PI3K/AKT pathway in breast cancer cells. Oncol Lett 15: 5952-5958, 2018.

30. Hong M, Wang N, Tan HY, Tsao SW and Feng Y: MicroRNAs and Chinese medicinal herbs: New possibilities in cancer therapy. Cancers (Basel) 7: 1643-1657, 2015.

31. Luo H, Vong CT, Chen H, Gao Y, Lyu P, Qiu L, Zhao M, Liu Q, Cheng Z, Zou J, et al: Naturally occurring anti-cancer compounds: Shining from Chinese herbal medicine. Chin Med 14: 48, 2019.

32. Kim HP, Son KH, Chang HW and Kang SS: Anti-inflammatory plant flavonoids and cellular action mechanisms. J Pharmacol Sci 96: 229-245, 2004

33. Lin L and Zakeri ZF: Apoptosis in development. Methods Mol Biol 136: 107-113, 2000

34. Weng D, Marty-Roix R, Ganesan S, Proulx MK, Vladimer GI, Kaiser WJ, Mocarski ES, Pouliot K, Chan FK, Kelliher MA, et al: Caspase- 8 and RIP kinases regulate bacteria-induced innate immune responses and cell death. Proc Natl Acad Sci USA 111: 7391-7396, 2014

35. Pistritto G, Trisciuoglio D, Ceci C, Garufi A and D'Orazi G: Apoptosis as anticancer mechanism: Function and dysfunction of its modulators and targeted therapeutic strategies. Aging (Albany NY) 8: 603-619, 2016.

36. Elmore S: Apoptosis: A review of programmed cell death. Toxicol Pathol 35: 495-516, 2007.

37. Brown R: The bcl-2 family of proteins. Br Med Bull 53: 466-477, 1997.

38. Roset R, Ortet L and Gil-Gomez G: Role of Bcl-2 family members on apoptosis: What we have learned from knock-out mice. Front Biosci 12: 4722-4730, 2007.

39. Zou J, Ardecky R, Pinkerton AB, Sergienko E, Su Y, Stonich D, Curpan RF, Simons PC, Zhai D, Diaz P, et al: Selective Bcl-2 inhibitor probes: In: Probe reports from the NIH molecular libraries program. Bethesda (MD): National Center for Biotechnology Information (US), 2010. https://www.ncbi.nlm. nih.gov/books/NBK133437/. Accessed October 31, 2011.

40. Burlacu A: Regulation of apoptosis by $\mathrm{Bcl}-2$ family proteins. J Cell Mol Med 7: 249-257, 2003

41. Schindler CK, Shinoda S, Simon RP and Henshall DC: Subcellular distribution of Bcl-2 family proteins and 14-3-3 within the hippocampus during seizure-induced neuronal death in the rat. Neurosci Lett 356: 163-166, 2004.

42. Tan KO, Fu NY, Sukumaran SK, Chan SL, Kang JH, Poon KL, Chen BS and Yu VC: MAP-1 is a mitochondrial effector of Bax. Proc Natl Acad Sci USA 102: 14623-14628, 2005.

43. Bruckheimer EM, Cho SH, Sarkiss M, Herrmann J and McDonnell TJ: The Bcl-2 gene family and apoptosis. Adv Biochem Eng Biotechnol 62: 75-105, 1998.

44. Tsukahara S, Yamamoto S, Tin-Tin-Win-Shwe, Ahmed S, Kunugita N, Arashidani K and Fujimaki H: Inhalation of low-level formaldehyde increases the $\mathrm{Bcl}-2 / \mathrm{Bax}$ expression ratio in the hippocampus of immunologically sensitized mice. Neuroimmunomodulation 13: 63-68, 2006. 
45. Pan Y, Ye C, Tian Q, Yan S, Zeng X, Xiao C, Wang L and Wang H: miR-145 suppresses the proliferation, invasion and migration of NSCLC cells by regulating the BAX/BCL-2 ratio and the caspase-3 cascade. Oncol Lett 15: 4337-4343, 2018.

46. Walensky LD: BCL-2 in the crosshairs: Tipping the balance of life and death. Cell Death Differ 13: 1339-1350, 2006.

47. Hastak K, Gupta S, Ahmad N, Agarwal MK, Agarwal ML and Mukhtar H: Role of p53 and NF-kappaB in epigallocatechin-3-gallate-induced apoptosis of LNCaP cells. Oncogene 22 4851-4859, 2003.

48. Lin HH, Chen JH, Huang CC and Wang CJ: Apoptotic effect of 3,4-dihydroxybenzoic acid on human gastric carcinoma cells involving JNK/p38 MAPK signaling activation. Int J Cancer 120: 2306-2316, 2007.
49. Kinnally KW and Antonsson B: A tale of two mitochondrial channels, MAC and PTP, in apoptosis. Apoptosis 12: 857-868, 2007.

50. Skulachev VP: Role of uncoupled and non-coupled oxidations in maintenance of safely low levels of oxygen and its one-electron reductants. Q Rev Biophys 29: 169-202, 1996.

This work is licensed under a Creative Commons Attribution-NonCommercial-NoDerivatives 4.0 International (CC BY-NC-ND 4.0) License. 